\title{
Bio-Efficacy of Imazethapyr in Rainfed Greengram [Vigna radiata (L.) Wilczek]
}

\author{
R Mohan Kumar ${ }^{1 *}$ and SM Hiremath ${ }^{2 *}$ \\ ${ }^{1}$ Scientist (Agronomy), AICRP-Castor, India \\ ${ }^{2}$ Department of Agronomy, University of Agricultural Sciences, India
}

Submission: August 11, 2018; Published: October 12, 2018

"Corresponding author: R Mohan Kumar, Scientist (Agronomy), AICRP-Castor, ZARS, GKVK, UAS-Bangalore (Karnataka), India, Email: mohankumarr@uasbangalore.edu.in

\begin{abstract}
A field experiment was conducted during kharif season of 2012 and 2013 in deep black soil at Main Agricultural Research Station Farm, University of Agricultural Sciences, Dharwad, Karnataka to find out the bio-efficacy of imazethapyr against weeds of greengram. Among the weed management treatments weed free check and standard check recorded significantly lower number weeds, total weed dry weight lower weed index. On the contrary these two treatments recorded higher weed control efficiency and total dry matter production of greengram. However,

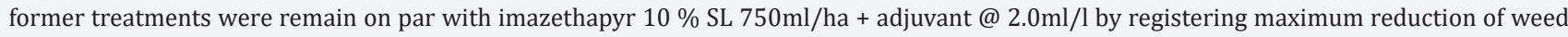
population, weed dry weight ( 5.12 and $10.4 \mathrm{~g} / \mathrm{m} 2$ during 2012 and 2013, respectively) and the highest weed control efficiency (89.55 and 78.77 \% during 2012 and 2013, respectively). Early post emergent application of imazethapyr applied at $10 \%$ SL 750ml/ha + adjuvant @ 2.0ml/1 recorded significantly higher plant height, LAI, total dry matter production and number of pods per plant, number of seeds per pod, seed and haulm yield. This was closely followed by the treatment with imazethapyr $10 \% \mathrm{SL} 750 \mathrm{ml} /$ ha. Weedy check treatment produced lowest yield of greengram.
\end{abstract}

Keywords: Greengram; Imazethapyr; Weeds; Weed control efficiency and Weed Index.

\section{Introduction}

Pulses are inseparable ingredients of vegetarian diet, and one of the cheapest weapons for combating the malnutrition problem by supplying dietary protein to the people. India contributes 27.65 per cent to the global pulse production and holds 35.2 per cent of the world's pulse acreage. Inspite of being the largest producer in the world, our country has to import pulses to the tune of two million tonnes every year to meet its domestic requirement, the increment in the production being not able to maintain the pace with population growth [1]. Among the grain legumes, greengram can be grown throughout the year, however, cultivation in rainy season is the common practice under rainfed situation. In India, greengram ranks third after chickpea and pigeon pea among the pulses in respect of production. In general weed infestation is saver during rainy season leads to drastic yield loss. One of the major constraints in greengram production is weed competition. The loss of greengram yield due to weeds ranges from 65.4 per cent to 79.0 per cent $[2,3]$. Imazethapyr is an imidazolinone herbicides applied as pre-emergence and early-post emergence for control of annual grass, broad-leaf weeds and perennial sedges in pulses. It is being a broad-spectrum herbicide found superior against all types of weeds. Since work on optimization of its dosage as early post emergence herbicides is a need of the hour. Further, during rainy season applied herbicide formulation subjected in to losses because of rainfall in such situation usage of adjuvant is the novel option to achieve desirable efficacy of the applied herbicide and work on these aspects are meager. Hence, an attempt was been made to evaluate the efficacy of imazethapyr at varied dosage.

\section{Material and Methods}

A field experiment was conducted to study the bio-efficacy of early post emergence herbicide Imazethapyr at various dosage in comparison with and Quizalofop - Ethyl 5 \% EC @1000ml/ha, standard check and weed free check against weeds in greengram and the impact different weeds management on growth and yield of greengram cultivar Nirmal was carried out at Main Agriculture Research Station Farm, University of Agricultural Sciences, Dharwad Karnataka during the kharif season 2012 and 2013. The soil of experimental site was clayey with $\mathrm{pH} 7.8$, having available nitrogen $230 \mathrm{~kg} / \mathrm{ha}$, available phosphorous $19.15 \mathrm{~kg} / \mathrm{ha}$, available potassium $303 \mathrm{~kg} / \mathrm{ha}$ and organic carbon $4.0 \mathrm{~g} / \mathrm{kg}$. The experiment was laid out in randomized complete block design having three replications. The treatment comprised of three doses of imazethapyr (500, 650 and $750 \mathrm{ml} / \mathrm{ha}$ ) with and without adjuvant, quizalofop - ethyl 5 \% EC @1000ml/ha, weedy check, standard check and weed free check. Herbicides application was done using a Knapsack sprayer equipped with a Flat-fan nozzle. The N, P and $\mathrm{K}$ was applied through urea, diammonium phosphate and muriate of potash as basal at sowing. The required plant population ( $30 \mathrm{~cm}$ row to row and $10 \mathrm{~cm}$ plant to plant) was maintained by thinning plants 20 days after sowing. The crop was raised under rainfed condition with recommended package of practices for the 
Northern Transitional Zone of Karnataka [4]. The total rainfall received during the crop growth was 148.4 and $40.5 \mathrm{~mm}$ in 7 and 2 rainy days during 2009-10, 2010-11, respectively.

\section{Results and Discussion}

\section{Weed flora of experimental site}

Weed flora noticed in experimental area consisted of grasses, broad leaved weeds and sedges. The important grassy weeds noticed were Cynodon dactylon, Chloris barbata, Dinebra retroflexa and Echinochloa colona. Broad leaved weeds noticed were Parthenium hysterophorus, Mollugo spp., Euphorbia hirta, Portulaca oleracea and Amaranthus viridis and sedge like Cyperus rotundus were common.

\section{Effect on grassy weeds}

Significantly lower number of grassy weeds were noticed in weed free check (0.71 and 1.41 at 40 and 60 DAS, respectively during 2012 and 1.22 and 1.41 at 40 and 60DAS during 2013) and standard check (2.74 and 2.65 at 40 and 60DAS, respectively during 2012 and 2.24 and 2.91 at 40 and 60DAS, respectively during 2013) (Table 1). Among the herbicides, Imazethapyr $10 \%$ SL at $625 \mathrm{ml}$ and Imazethapyr $10 \%$ SL at $750 \mathrm{ml}$ per hectare with or without adjuvant effectively controlled the total grassy weed (Cynodon dactylon, Dinebra retroflexa, Chloris barbata and Echinochloa colona) and was reflected by recording higher species wise weed control efficiency during both the years of experimentation. Application of imazethapyr was effective against annual grassy weeds and perennial sedges were earlier reported by Goud et al. [5] However, grassy weed control efficiency of Imazethapyr $10 \%$ SL 750ml/ha + Adjuvant @ 2.0ml/l of water was remain comparable with that of Quizalofop-Ethyl $5 \%$ EC $@ 1000 \mathrm{ml} /$ ha (Table 2 and 3). These results are in agreement with the findings of Tiwari et al. [6].

Table 1: Total number of weeds per square meter at different stages of greengram under different weed management practices.

\begin{tabular}{|c|c|c|c|c|c|c|c|c|c|c|c|c|}
\hline \multirow[b]{2}{*}{ Treatments } & \multicolumn{2}{|c|}{ Grasses 2012} & \multicolumn{2}{|c|}{ Grasses 2013} & \multicolumn{2}{|c|}{ BLW 2012} & \multicolumn{2}{|c|}{ BLW 2013} & \multicolumn{2}{|c|}{ Sedges 2012} & \multicolumn{2}{|c|}{ Sedges 2012} \\
\hline & 40 DAS & 60 DAS & 40 DAS & 60 DAS & 40 DAS & 60 DAS & $\begin{array}{c}40 \\
\text { DAS }\end{array}$ & 60 DAS & 40 DAS & 60 DAS & 40 DAS & 60 DAS \\
\hline $\begin{array}{l}\text { T1 - Imazethapyr } 10 \\
\% \text { SL 500ml/ha }\end{array}$ & $\begin{array}{c}* 3.87 \\
(14.50)\end{array}$ & $\begin{array}{c}4.79 \\
(22.50)\end{array}$ & $\begin{array}{c}3.81 \\
(14.00)\end{array}$ & $\begin{array}{c}4.30 \\
(18.00)\end{array}$ & $\begin{array}{c}2.83 \\
(7.50)\end{array}$ & $\begin{array}{c}3.67 \\
(13.00)\end{array}$ & $\begin{array}{c}2.74 \\
(7.00)\end{array}$ & $\begin{array}{c}3.39 \\
(11.00)\end{array}$ & $\begin{array}{c}2.00 \\
(3.50)\end{array}$ & $\begin{array}{c}2.93 \\
(8.00)\end{array}$ & $\begin{array}{c}1.87 \\
(3.00)\end{array}$ & $\begin{array}{c}2.65 \\
(6.50)\end{array}$ \\
\hline $\begin{array}{l}\text { T2 - Imazethapyr } 10 \\
\% \text { SL } 625 \mathrm{ml} / \mathrm{ha}\end{array}$ & $\begin{array}{c}3.46 \\
(11.50)\end{array}$ & $\begin{array}{c}4.47 \\
(19.50)\end{array}$ & $\begin{array}{c}3.46 \\
(11.50)\end{array}$ & $\begin{array}{c}4.18 \\
(17.00)\end{array}$ & $\begin{array}{c}2.45 \\
(5.50)\end{array}$ & $\begin{array}{c}3.24 \\
(10.00)\end{array}$ & $\begin{array}{c}2.34 \\
(5.00)\end{array}$ & $\begin{array}{c}3.00 \\
(8.50)\end{array}$ & $\begin{array}{c}1.58 \\
(2.00)\end{array}$ & $\begin{array}{c}2.74 \\
(7.00)\end{array}$ & $\begin{array}{c}1.41 \\
(1.50)\end{array}$ & $\begin{array}{c}2.55 \\
(6.00)\end{array}$ \\
\hline $\begin{array}{l}\text { T3 - Imazethapyr } 10 \\
\% \text { SL 750ml/ha }\end{array}$ & $\begin{array}{c}3.16 \\
(9.50)\end{array}$ & $\begin{array}{c}4.30 \\
(18.00)\end{array}$ & $\begin{array}{c}3.39 \\
(11.00)\end{array}$ & $\begin{array}{c}4.06 \\
(16.00)\end{array}$ & $\begin{array}{c}2.83 \\
(7.50)\end{array}$ & $\begin{array}{c}3.46 \\
(11.50)\end{array}$ & $\begin{array}{c}2.74 \\
(7.00)\end{array}$ & $\begin{array}{c}3.16 \\
(9.50)\end{array}$ & $\begin{array}{c}1.41 \\
(1.50)\end{array}$ & $\begin{array}{c}2.34 \\
(5.00)\end{array}$ & $\begin{array}{c}1.00 \\
(0.50)\end{array}$ & $\begin{array}{c}2.34 \\
(5.00)\end{array}$ \\
\hline $\begin{array}{c}\text { T4 - Imazethapyr } \\
10 \% \text { SL 500ml/ha } \\
\text { +Adjuvant }\end{array}$ & $\begin{array}{c}3.39 \\
(11.00)\end{array}$ & $\begin{array}{c}4.53 \\
(20.00)\end{array}$ & $\begin{array}{c}3.39 \\
(11.00)\end{array}$ & $\begin{array}{c}4.18 \\
(17.00)\end{array}$ & $\begin{array}{c}2.74 \\
(7.00)\end{array}$ & $\begin{array}{c}3.32 \\
(10.50)\end{array}$ & $\begin{array}{c}2.83 \\
(7.50)\end{array}$ & $\begin{array}{c}3.53 \\
(12.00)\end{array}$ & $\begin{array}{c}2.45 \\
(5.50)\end{array}$ & $\begin{array}{c}2.00 \\
(3.50)\end{array}$ & $\begin{array}{c}2.00 \\
(3.50)\end{array}$ & $\begin{array}{c}2.24 \\
(4.50)\end{array}$ \\
\hline $\begin{array}{c}\text { T5 - Imazethapyr } 10 \\
\% \text { SL 625ml/ha + } \\
\text { Adjuvant }\end{array}$ & $\begin{array}{c}3.08 \\
(9.00)\end{array}$ & $\begin{array}{c}3.67 \\
(13.00)\end{array}$ & $\begin{array}{c}3.08 \\
(9.00)\end{array}$ & $\begin{array}{c}3.81 \\
(14.00)\end{array}$ & $\begin{array}{c}2.45 \\
(5.50)\end{array}$ & $\begin{array}{c}3.24 \\
(10.00)\end{array}$ & $\begin{array}{c}2.74 \\
(7.00)\end{array}$ & $\begin{array}{c}3.39 \\
(11.00)\end{array}$ & $\begin{array}{c}1.22 \\
(1.00)\end{array}$ & $\begin{array}{c}2.24 \\
(4.50)\end{array}$ & $\begin{array}{c}1.58 \\
(2.00)\end{array}$ & $\begin{array}{c}2.24 \\
(4.50)\end{array}$ \\
\hline $\begin{array}{c}\text { T6 - Imazethapyr } \\
10 \% \text { SL 750ml/l + } \\
\text { Adjuvant }\end{array}$ & $\begin{array}{c}3.39 \\
(11.00)\end{array}$ & $\begin{array}{c}3.60 \\
(12.50)\end{array}$ & $\begin{array}{c}2.91 \\
(8.00)\end{array}$ & $\begin{array}{c}3.87 \\
(14.50)\end{array}$ & $\begin{array}{l}2.45 \\
(5.50)\end{array}$ & $\begin{array}{c}3.00 \\
(8.50)\end{array}$ & $\begin{array}{c}2.55 \\
(6.00)\end{array}$ & $\begin{array}{c}3.16 \\
(9.50)\end{array}$ & $\begin{array}{c}1.22 \\
(1.00)\end{array}$ & $\begin{array}{c}1.73 \\
(2.50)\end{array}$ & $\begin{array}{c}1.22 \\
(1.00)\end{array}$ & $\begin{array}{c}1.73 \\
(2.50)\end{array}$ \\
\hline $\begin{array}{c}\text { T7 - Quizalofop - } \\
\text { Ethyl } 5 \text { \% EC @1000 } \\
\text { ml/ha }\end{array}$ & $\begin{array}{c}3.08 \\
(9.00)\end{array}$ & $\begin{array}{c}3.60 \\
(12.50)\end{array}$ & $\begin{array}{l}3.08 \\
(9.00)\end{array}$ & $\begin{array}{c}3.94 \\
(15.00)\end{array}$ & $\begin{array}{c}3.08 \\
(9.00)\end{array}$ & $\begin{array}{c}3.81 \\
(14.00)\end{array}$ & $\begin{array}{c}3.08 \\
(9.00)\end{array}$ & $\begin{array}{c}3.74 \\
(13.50)\end{array}$ & $\begin{array}{l}1.58 \\
(2.00)\end{array}$ & $\begin{array}{c}2.74 \\
(7.00)\end{array}$ & $\begin{array}{c}1.22 \\
(1.00)\end{array}$ & $\begin{array}{c}2.74 \\
(7.00)\end{array}$ \\
\hline $\begin{array}{c}\text { T8 - Standrad check } \\
(2 \mathrm{IC}+2 \mathrm{HW})\end{array}$ & $\begin{array}{c}2.74 \\
(7.00)\end{array}$ & $\begin{array}{c}2.65 \\
(6.50)\end{array}$ & $\begin{array}{c}2.24 \\
(4.50)\end{array}$ & $\begin{array}{c}2.91 \\
(8.00)\end{array}$ & $\begin{array}{c}2.12 \\
(4.00)\end{array}$ & $\begin{array}{c}2.65 \\
(6.50)\end{array}$ & $\begin{array}{l}1.87 \\
(3.00)\end{array}$ & $\begin{array}{c}2.65 \\
(6.50)\end{array}$ & $\begin{array}{c}1.22 \\
(1.00)\end{array}$ & $\begin{array}{c}1.41 \\
(1.50)\end{array}$ & $\begin{array}{c}1.22 \\
(1.00)\end{array}$ & $\begin{array}{c}1.41 \\
(1.50)\end{array}$ \\
\hline T9 - Weedy check & $\begin{array}{c}5.19 \\
(26.50)\end{array}$ & $\begin{array}{c}6.78 \\
(45.50)\end{array}$ & $\begin{array}{c}5.57 \\
(30.50)\end{array}$ & $\begin{array}{c}5.83 \\
(33.50)\end{array}$ & $\begin{array}{c}3.81 \\
(14.00)\end{array}$ & $\begin{array}{c}4.24 \\
(17.50)\end{array}$ & $\begin{array}{c}3.81 \\
(14.00)\end{array}$ & $\begin{array}{c}4.30 \\
(18.00)\end{array}$ & $\begin{array}{c}3.32 \\
(10.50)\end{array}$ & $\begin{array}{c}4.06 \\
(16.00)\end{array}$ & $\begin{array}{c}2.65 \\
(6.50)\end{array}$ & $\begin{array}{c}3.87 \\
(14.50)\end{array}$ \\
\hline T10 - Weed free & $\begin{array}{c}0.71 \\
(0.00)\end{array}$ & $\begin{array}{c}1.41 \\
(1.50)\end{array}$ & $\begin{array}{c}1.22 \\
(1.00)\end{array}$ & $\begin{array}{c}1.41 \\
(1.50)\end{array}$ & $\begin{array}{c}1.41 \\
(1.50)\end{array}$ & $\begin{array}{c}1.58 \\
(2.00)\end{array}$ & $\begin{array}{l}1.00 \\
(0.50)\end{array}$ & $\begin{array}{c}1.41 \\
(1.50)\end{array}$ & $\begin{array}{l}1.58 \\
(2.00)\end{array}$ & $\begin{array}{c}1.87 \\
(3.00)\end{array}$ & $\begin{array}{l}1.00 \\
(0.50)\end{array}$ & $\begin{array}{c}1.22 \\
(1.00)\end{array}$ \\
\hline S. Em \pm & 0.03 & 0.04 & 0.03 & 0.03 & 0.02 & 0.02 & 0.02 & 0.02 & 0.02 & 0.43 & 0.02 & 0.02 \\
\hline$C D(p=0.05)$ & 0.07 & 0.08 & 0.06 & 0.06 & 0.04 & 0.04 & 0.04 & 0.04 & 0.04 & 0.91 & 0.03 & 0.04 \\
\hline
\end{tabular}

Note * $(x+0.5)^{1 / 2}$ transformed data; figures in the parentheses indicate original values.

Table 2: Species wise weed control efficiency in green gram as influenced by different weed management practices at 60 DAS during 2012.

\begin{tabular}{|c|c|c|c|c|c|c|c|c|c|c|c|c|c|c|}
\hline \multirow{2}{*}{ Treatments } & \multicolumn{6}{|c|}{ Grasses } & \multicolumn{6}{|c|}{ BLW } & \multicolumn{2}{|c|}{ Sedge } \\
\hline & Cd & $\mathrm{Dr}$ & $\mathrm{Cb}$ & Ec & Others & Total & $\mathbf{P h}$ & Av & Po & Ms & Eh & Total & $\mathrm{Cr}$ & Total \\
\hline $\begin{array}{c}\text { T1-Imazethapyr } 10 \% \mathrm{SL} \\
500 \mathrm{ml} / \mathrm{ha}\end{array}$ & 57.14 & 47.83 & 53.85 & 60 & 50 & 55.45 & 25 & 40 & 55.56 & 33.33 & 100 & 46.94 & 50 & 50 \\
\hline $\begin{array}{c}\text { T2-Imazethapyr } 10 \% \mathrm{SL} \\
625 \mathrm{ml} / \mathrm{ha}\end{array}$ & 59.18 & 65.22 & 69.23 & 80 & 75 & 61.39 & 50 & 70 & 55.56 & 33.33 & 92.86 & 59.18 & 56.25 & 56.25 \\
\hline
\end{tabular}


Annals of Reviews and Research

\begin{tabular}{|c|c|c|c|c|c|c|c|c|c|c|c|c|c|c|}
\hline $\begin{array}{c}\text { T3-Imazethapyr } 10 \% \mathrm{SL} \\
750 \mathrm{ml} / \mathrm{ha}\end{array}$ & 65.31 & 65.22 & 61.54 & 40 & 100 & 64.36 & 37.5 & 60 & 66.67 & 33.33 & 85.71 & 53.06 & 68.75 & 68.75 \\
\hline $\begin{array}{c}\text { T4- Imazethapyr } 10 \% \mathrm{SL} \\
500 \mathrm{ml} / \mathrm{ha}+\text { Adjuvant @ } \\
2.0 \mathrm{ml} / \mathrm{l} \text { of water }\end{array}$ & 61.22 & 56.52 & 46.15 & 60 & 100 & 60.4 & 56.25 & 50 & 77.78 & 33.33 & 78.57 & 57.14 & 78.13 & 78.13 \\
\hline $\begin{array}{l}\text { T5- Imazethapyr } 10 \% \mathrm{SL} \\
625 \mathrm{ml} / \mathrm{ha}+\text { Adjuvant } @ \\
2.0 \mathrm{ml} / \mathrm{l} \text { of water }\end{array}$ & 79.59 & 78.26 & 61.54 & 60 & 50 & 74.26 & 56.25 & 70 & 77.78 & 33.33 & 71.43 & 59.18 & 71.88 & 71.88 \\
\hline $\begin{array}{l}\text { T6- Imazethapyr } 10 \% \text { SL } \\
750 \mathrm{ml} / \mathrm{ha}+\text { Adjuvant @ } \\
2.0 \mathrm{ml} / \mathrm{l} \text { of water }\end{array}$ & 81.63 & 78.26 & 69.23 & 40 & 75 & 75.25 & 75 & 50 & 77.78 & 50 & 78.57 & 65.31 & 84.38 & 84.38 \\
\hline $\begin{array}{l}\text { T7-Quizalofop-Ethyl } 5 \text { \% EC } \\
\text { @1000ml/ha }\end{array}$ & 81.63 & 73.91 & 61.54 & 70 & 50 & 75.25 & 18.75 & 50 & 33.33 & 66.67 & 85.71 & 42.86 & 56.25 & 56.25 \\
\hline $\begin{array}{c}\text { T8-Standrad check (2 } \\
\text { IC+2HW) }\end{array}$ & 91.84 & 86.96 & 76.92 & 80 & 75 & 87.13 & 87.5 & 70 & 77.78 & 0 & 100 & 73.47 & 90.63 & 90.63 \\
\hline T9-Weedy check & - & - & - & - & - & - & - & - & - & - & - & - & - & - \\
\hline T10-Weed free & 100 & 91.3 & 100 & 100 & 75 & 97.03 & 100 & 80 & 100 & 66.67 & 100 & 91.84 & 81.25 & 81.25 \\
\hline
\end{tabular}

Table 3: Species wise weed control efficiency in green gram as influenced by different weed management practices at 60 DAS during 2013.

\begin{tabular}{|c|c|c|c|c|c|c|c|c|c|c|c|c|c|c|}
\hline \multirow{2}{*}{ Treatments } & \multicolumn{6}{|c|}{ Grasses } & \multicolumn{6}{|c|}{ BLW } & \multicolumn{2}{|c|}{ Sedge } \\
\hline & Cd & Dr & $\mathbf{C b}$ & Ec & Others & Total & $\mathbf{P h}$ & Av & Po & Ms & Eh & Total & $\mathrm{Cr}$ & Total \\
\hline $\begin{array}{l}\text { T1-Imazethapyr } 10 \% \\
\text { SL 500ml/ha }\end{array}$ & 52.63 & 41.18 & 50 & 50 & 100 & 50.68 & 52.94 & 40 & 42.86 & 15.33 & 100 & 52.41 & 55.17 & 55.17 \\
\hline $\begin{array}{l}\text { T2-Imazethapyr } 10 \% \\
\text { SL } 625 \mathrm{ml} / \mathrm{ha}\end{array}$ & 55.26 & 41.18 & 50 & 75 & 50 & 53.42 & 70.59 & 70 & 42.86 & 22.33 & 88.24 & 63.59 & 58.62 & 58.62 \\
\hline $\begin{array}{l}\text { T3-Imazethapyr } 10 \% \\
\text { SL 750ml/ha }\end{array}$ & 65.79 & 52.94 & 37.5 & 25 & 100 & 56.16 & 64.71 & 60 & 57.14 & 33.33 & 76.47 & 60.82 & 65.52 & 65.52 \\
\hline $\begin{array}{c}\text { T4- Imazethapyr } 10 \% \\
\text { SL } 500 \mathrm{ml} / \mathrm{ha}+\text { Adju- } \\
\text { vant @ } 2.0 \mathrm{ml} / \mathrm{l} \text { of water }\end{array}$ & 63.16 & 47.06 & 12.5 & 50 & 100 & 53.42 & 58.82 & 50 & 28.57 & 33.33 & 64.71 & 50.52 & 68.97 & 68.97 \\
\hline $\begin{array}{l}\text { T5- Imazethapyr } 10 \% \\
\text { SL } 625 \mathrm{ml} / \mathrm{ha}+\text { Adju- } \\
\text { vant @ } 2.0 \mathrm{ml} / \mathrm{l} \text { of water }\end{array}$ & 71.05 & 64.71 & 37.5 & 50 & 0 & 61.64 & 58.82 & 70 & 42.86 & 75.33 & 52.94 & 59.84 & 68.97 & 68.97 \\
\hline $\begin{array}{c}\text { T6- Imazethapyr } 10 \% \\
\text { SL } 750 \mathrm{ml} / \mathrm{ha}+\text { Adju- } \\
\text { vant @ } 2.0 \mathrm{ml} / \mathrm{l} \text { of water }\end{array}$ & 73.68 & 52.94 & 37.5 & 25 & 100 & 60.27 & 70.59 & 50 & 57.14 & 75.33 & 64.71 & 63.96 & 82.76 & 82.76 \\
\hline $\begin{array}{l}\text { T7-Quizalofop-Ethyl } 5 \text { \% } \\
\text { EC @1000ml/ha }\end{array}$ & 68.42 & 64.71 & 12.5 & 62.5 & 0 & 58.9 & 29.41 & 50 & 14.29 & 66.67 & 76.47 & 44.33 & 51.72 & 51.72 \\
\hline $\begin{array}{c}\text { T8-Standrad check }(2 \\
\text { IC+2HW) }\end{array}$ & 84.21 & 76.47 & 62.5 & 75 & 50 & 78.08 & 88.24 & 70 & 71.43 & 0 & 100 & 73.2 & 89.66 & 89.66 \\
\hline T9-Weedy check & - & - & - & - & - & - & - & - & - & - & - & - & - & - \\
\hline T10-Weed free & 92.11 & 100 & 100 & 100 & 100 & 95.89 & 94.12 & 80 & 100 & 100 & 100 & 93.81 & 93.1 & 93.1 \\
\hline
\end{tabular}

Cd: Cynodon dactylon Dr: Dinebra retroflexa Cb: Chloris barbata Ec: Echinochloa colona

Ph: Parthenium hysterophorus, Av: Amaranthus viridis, Po: Partulca oleracea, Eh: Euphorbia hirta, M spp: Mullugo spp

\section{Effect on broad leaf weeds (BLW)}

At 40 and 60 days after sowing weed free check (1.41 and 1.58 at 40 and 60DAS, respectively during 2012 and 1.00 and 1.41 at 40 and 60 DAS, respectively during 2013 and standard check (2.12 and 2.65 at 40 and 60DAS, respectively and 1.87 and 2.65 at 40 and 60DAS, respectively during 2013) treatments recorded significantly lower broad-leaved weeds. Imazethapyr $10 \% \mathrm{SL}$ at $625 \mathrm{ml} / \mathrm{ha}+$ adjuvant ( 2.45 and 3.24 at 40 and 60DAS, respectively during 2012 and 2.74 and 3.39 at 40 and 60DAS, respectively during 2013) and 750ml/ha + adjuvant (2.45 and 3.00 during 40 and 60 DAS, respectively during 2012 and 2.55 and 3.16 at 40 and 60 DAS, respectively during 2013) remained on par with former treatments. Further, Imazethapyr $10 \%$ SL at 625 and $750 \mathrm{ml}$ per ha alone was effective against the broad-leaved weeds such as Amaranthus viridis, Portulaca oleracea, Euphorbia hirta and Mollugo spp during both the year of experimentation. However, it was not effective against Parthenium. Significantly higher broadleaved weeds were noticed in Quizalofop-Ethyl 5\% EC @ 1000ml/ 
ha and weedy check Tiwari et al. [6] Application of imazethapyr at $0.10 \mathrm{~kg} / \mathrm{ha}$ controlled the weeds most effectively than other herbicides in summer irrigated blackgram [7].

\section{Effect on sedge}

During both the years of study at 40 and 60 days after sowing, significantly lower sedge weeds were noticed in weed free check (1.58 and 1.87 during 40 and 60 DAS, respectively) and standard check (1.22 and 1.41 during 40 and 60 DAS, respectively) treatments. Among the different doses of Imazathapyr, lower sedge number was noticed with Imazathapyr @ 750ml + adjuvant followed by Imazathapyr @ 625ml + adjuvant at 40 and 60 DAS. $[5,8]$ reported that imazethapyr controlled Cyperus rotundus more effectively when applied to weeds at 5 to $20 \mathrm{~cm}$ tall compared with weeds of $30 \mathrm{~cm}$ tall. Hence, early post emergence application of imazethapyr was the excellent chemical option against perineal sedges. Data on species wise weed control efficiency reveled that, Imazethapyr $10 \%$ SL 750ml/ha + Adjuvant @ 2.0ml/l of water found superior in controlling Cyperus rotundus.

\section{Grassy weed dry matter production}

Observation recorded on weed dry matter at 60 days after sowing of the crop revealed that significantly lower weed dry matter of grassy weeds were recorded in Imazethapyr 10\% SL $750 \mathrm{ml} /$ ha with $2 \mathrm{ml}$ of MSO adjuvant (1.79 and $4.93 \mathrm{gm}^{-2}$ during 2012 and 2013, respectively) compared to Imazethapyr 10\% SL $500 \mathrm{ml} /$ ha (with or without adjuvant) (Table 1). However,
Imazethapyr $10 \% \mathrm{SL}$ at $750 \mathrm{ml} /$ ha alone $\left(2.38\right.$ and $5.69 \mathrm{gm}^{-2}$ during 2012 and 2013, respectively), Imazethapyr 10\% SL 625ml with or without adjuvant, Quizalofop-p-ethyl 5\% EC @1000ml/ha (2.68 and 5.37gm-2 during 2012 and 2013, respectively), standard check (2IC + 2HW) (2.68 and 2.85 gm $^{-2}$ during 2012 and 2013, respectively) and weed free check ( 0.66 and $0.70 \mathrm{gm}^{-2}$ during 2012 and 2013, respectively) remained on par with former treatments. Significantly higher dry matter accumulation in grassy weeds was recorded in weedy check.

\section{Broad Leaved Weeds Weed Dry Matter Production}

Data on dry matter of broad leaved weed at 60 days after sowing (Table 4) revealed that significantly lower dry matter accumulation was noticed in weed free check $(0.69$ and $0.0 .87 \mathrm{gm}$ ${ }^{2}$ during 2012 and 2013, respectively), standard check (3.31 and $3.41 \mathrm{gm}^{-2}$ during 2012 and 2013, respectively) and Imazethapyr $10 \% \mathrm{SL}$ at 750 with $2 \mathrm{ml}$ of MSO adjuvant (2.98 and $4.99 \mathrm{gm}^{-2}$ during 2012 and 2013, respectively) than weedy check (23.16 and $25.70 \mathrm{gm}^{-2}$ during 2012 and 2013, respectively) on account of lower number of weed and higher species wise weed control efficiency. On the contrary, Imazethapyr 10\% SL $500 \mathrm{ml} / \mathrm{ha}$ with adjuvant (6.62 and 6.30 $\mathrm{gm}^{-2}$ during 2012 and 2013, respectively) or without $2 \mathrm{ml}$ adjuvant (6.62 and $5.60 \mathrm{gm}^{-2}$ during 2012 and 2013, respectively) and Quizalofop-p-ethyl 5\% EC @1000ml/ha recorded significantly higher amount of dry matter accumulation in broad weeds (6.62 and 7.08gm ${ }^{-2}$ during 2012 and 2013, respectively).

Table 4: Weed dry weight, weed control efficiency and weed index in green gram as influenced by different weed management practices.

\begin{tabular}{|c|c|c|c|c|c|c|c|c|c|c|c|c|}
\hline \multirow{3}{*}{ Treatments } & \multicolumn{7}{|c|}{ Weed dry weight ( $\mathrm{g} \mathrm{m}$ - 2) [60 DAS] } & \multirow{2}{*}{\multicolumn{3}{|c|}{$\begin{array}{l}\text { Weed control efficiency } \\
\qquad \%)\end{array}$}} & \multirow{2}{*}{\multicolumn{2}{|c|}{ Weed index $(\%)$}} \\
\hline & \multicolumn{2}{|c|}{ Grasses } & \multicolumn{2}{|c|}{ BLW } & \multicolumn{2}{|c|}{ Sedges } & \multirow{2}{*}{\begin{tabular}{l|} 
Total \\
2012 \\
\end{tabular}} & & & & & \\
\hline & 2012 & 2013 & 2012 & 2013 & 2012 & 2013 & & 2013 & 2012 & 2013 & 2012 & 2013 \\
\hline $\begin{array}{r}\text { T1 - Imazethapyr } 10 \% \mathrm{SL} \\
500 \mathrm{ml} / \mathrm{ha}\end{array}$ & 6.84 & 6.07 & 6.62 & 5.6 & 0.83 & 1.43 & 14.28 & 13.11 & 70.18 & 74.04 & 44.43 & 44.4 \\
\hline $\begin{array}{r}\text { T2 - Imazethapyr } 10 \% \mathrm{SL} \\
625 \mathrm{ml} / \mathrm{ha}\end{array}$ & 5.35 & 5.66 & 4.63 & 4.46 & 0.65 & 1.32 & 10.64 & 11.44 & 77.95 & 78.3 & 32.79 & 24.41 \\
\hline $\begin{array}{r}\text { T3 - Imazethapyr } 10 \% \text { SL } \\
750 \mathrm{ml} / \mathrm{ha}\end{array}$ & 2.38 & 5.69 & 2.65 & 4.98 & 0.59 & 1.1 & 5.62 & 11.77 & 88.24 & 75.97 & 21.9 & 12.12 \\
\hline $\begin{array}{r}\text { T4 - Imazethapyr } 10 \% \mathrm{SL} \\
500 \mathrm{ml} / \mathrm{ha}+ \\
\text { Adjuvant @ } \\
2.0 \mathrm{ml} / \mathrm{l} \text { of } \\
\text { water }\end{array}$ & 5.36 & 5.93 & 6.62 & 6.3 & 0.42 & 0.99 & 12.38 & 13.21 & 75.06 & 74.05 & 28.83 & 33.3 \\
\hline $\begin{array}{r}\text { T5 - Imazethapyr } 10 \% \mathrm{SL} \\
625 \mathrm{ml} / \mathrm{ha}+ \\
\text { Adjuvant @ } \\
2.0 \mathrm{ml} / \mathrm{l} \text { of } \\
\text { water }\end{array}$ & 4.76 & 5.61 & 3.97 & 5.77 & 0.42 & 0.99 & 9.14 & 12.37 & 81.92 & 74.94 & 15.03 & 20.47 \\
\hline $\begin{array}{r}\text { T6 - Imazethapyr } 10 \% \mathrm{SL} \\
750 \mathrm{ml} / \mathrm{ha}+ \\
\text { Adjuvant @ } \\
2.0 \mathrm{ml} / \mathrm{l} \text { of } \\
\text { water }\end{array}$ & 1.79 & 4.93 & 2.98 & 4.99 & 0.36 & 0.55 & 5.12 & 10.47 & 89.55 & 78.77 & 6.72 & 10.54 \\
\hline $\begin{array}{r}\text { T7 - Quizalofop - Ethyl } 5 \% \\
\text { EC @1000 } \\
\mathrm{ml} / \mathrm{ha}\end{array}$ & 2.68 & 5.37 & 6.62 & 7.08 & 0.71 & 1.54 & 10.01 & 13.99 & 80.23 & 72.11 & 34.35 & 27.67 \\
\hline $\begin{array}{r}\text { T8 - Standrad check (2 } \\
\text { IC }+2 \mathrm{HW})\end{array}$ & 2.68 & 2.85 & 3.31 & 3.41 & 0.3 & 0.33 & 6.28 & 6.59 & 87.38 & 87.06 & 1.36 & 6.98 \\
\hline
\end{tabular}


Annals of Reviews and Research

\begin{tabular}{|c|c|c|c|c|c|c|c|c|c|c|c|c|}
\hline T9 - Weedy check & 25.58 & 26.61 & 23.16 & 25.7 & 2.95 & 3.19 & 51.69 & 55.5 & - & - & 47.79 & 55.76 \\
\hline T10 - Weed free & 0.66 & 0.7 & 0.69 & 0.87 & 0.71 & 0.22 & 2.03 & 0.92 & 96.97 & 98.62 & - & - \\
\hline S. Em. \pm & $\mathbf{1 . 6 7}$ & $\mathbf{5 . 6}$ & $\mathbf{2 . 6 8}$ & $\mathbf{1 . 1 5}$ & $\mathbf{0 . 4 8}$ & $\mathbf{0 . 5 3}$ & $\mathbf{4 . 4 9}$ & $\mathbf{4 . 9 8}$ & $\mathbf{4 . 1 3}$ & $\mathbf{4 . 4 2}$ & $\mathbf{1 . 1}$ & $\mathbf{2 . 4 7}$ \\
\hline CD (p=0.05) & $\mathbf{3 . 5}$ & $\mathbf{1 1 . 7 7}$ & $\mathbf{5 . 6 4}$ & $\mathbf{2 . 4 1}$ & $\mathbf{1 . 0 2}$ & $\mathbf{1 . 1 2}$ & $\mathbf{9 . 5 1}$ & $\mathbf{1 0 . 4 7}$ & $\mathbf{8 . 7 6}$ & $\mathbf{9 . 2 9}$ & $\mathbf{2 . 3 3}$ & $\mathbf{6 . 2 7}$ \\
\hline
\end{tabular}

\section{Sedges Weed Dry Matter Production}

At 60 days after sowing, Imazethapyr 10\% SL at all the levels with or without MSO adjuvant and Quizalofop-p-ethyl 5\% EC $@ 1000 \mathrm{ml} /$ ha recorded lower weed dry weight in sedge and were statistically on par with the standard check (2 IC $+2 \mathrm{HW}$ ). It was mainly due to lower sedge count with former treatments and was again reflected by recording higher sedge count efficiency. Significantly higher dry matter accumulation in sedges was noticed in weedy check.

\section{Weed control efficiency}

\section{Grasses}

Significantly higher weed control efficiency was noticed in weed free check (97.03\%) than lower levels of Imazethapyr (500 \& $625 \mathrm{ml} / \mathrm{ha}$ ) (Table 2). However, Imazethapyr 10\% SL $750 \mathrm{ml} /$ ha with MSO adjuvant (95.25\%), Imazethapyr 10\% SL 750ml/ ha (64.36 \%), Quizalofop-p-ethyl 5\% EC @1000ml/ha (75.25 \%) were found on par with former treatment. Significantly lower weed control efficiency was noticed in rest of lower doses of herbicides applied with or without adjuvant. The similar trend was noticed during 2013 (Table 3), wherein weed free check (95.89 $\%$ ), Imazethapyr 10\% SL 750ml/ha with MSO adjuvant (60.27\%), Imazethapyr 10\% SL 750ml/ha (56.16\%) and Quizalofop-p-ethyl $5 \%$ EC @ $1000 \mathrm{ml} /$ ha (58.90 \%) recorded higher weed control efficiency than other treatments. Present findings are in line with [9].

\section{Broad leaved weeds}

Significantly higher broad-leaved weed control efficiency was recorded in weed free check (91.84\%) and standard check (73.47 \%) (Table 2). Among the herbicides, Imazethapyr 10\% SL 625 and $750 \mathrm{ml} /$ ha both with or without $2 \mathrm{ml}$ MSO adjuvant per liter of water were significantly superior over Imazethapyr 10\% SL $500 \mathrm{ml} /$ ha with or without $2 \mathrm{ml} \mathrm{MSO}$ adjuvant and Quizalofop-Ethyl 5\% EC @1000ml/ha during 2012. Whereas, during 2013 (Table 3 ), weed free check excelled over other treatments. Among the herbicides, Imazethapyr $10 \%$ SL $750 \mathrm{ml}$ with or without adjuvant recorded significantly higher broad-leaved control efficiency and was on par with rest of the treatments except Quizalofop-Ethyl $5 \%$ EC @1000ml/ha (Table 3). It could be due to better control of broad-leaved weeds by Imazethapyr at higher doses.

\section{Sedge}

Significantly higher sedge weed control efficiency was noticed with Imazethapyr $10 \%$ SL $750 \mathrm{ml} /$ ha with and without MSO adjuvant. Whereas sedge weeds control efficiency was significantly lower in Imazethapyr $10 \%$ SL $500 \mathrm{ml} /$ ha over rest of the treatments. Similar, tread was noticed during both the years of experimentation.

\section{Weed index $(\%)$}

Weed index indicate the extent of yield reduction by weed infestation. During both the years of experimentation (2012 and 2013) significantly lower weed index was noticed in standard check treatment (1.36 and 6.98\% during 2012 and 2013, respectively). Among the herbicides, Imazethapyr 10\% SL 750 $\mathrm{ml} /$ ha with 2ml/l MSO adjuvant (6.72 and 10.54\% during 2012 and 2013, respectively) recorded lower weed index. Among the herbicides, significantly higher weed index was observed in Imazethapyr $10 \%$ SL $500 \mathrm{ml} /$ ha without $2 \mathrm{ml} /$ ha MSO adjuvant per liter of water (44.43 and 44.40\% during 2012 and 2013, respectively). Significantly higher weed control efficiency could be the causes for significantly lower weed index.

\section{Growth and Yield Attributes of Greengram}

Table 5: Growth, yield attributes, grain and haulm yield of green gram as influenced by different weed management practices (Pooled data of two years).

\begin{tabular}{|c|c|c|c|c|c|c|c|c|c|}
\hline Treatment & $\begin{array}{c}\text { Plant Height } \\
\text { at Harvest } \\
\text { (cm) }\end{array}$ & $\begin{array}{l}\text { LAI at } \\
45 \text { DAS }\end{array}$ & $\begin{array}{l}\text { TDMP } \\
\text { (g/ } \\
\text { plant) }\end{array}$ & $\begin{array}{l}\text { No. pods } \\
\text { per plant }\end{array}$ & $\begin{array}{c}\text { Number of } \\
\text { Seeds Per } \\
\text { pod }\end{array}$ & $\begin{array}{c}\text { Test } \\
\text { Weight }\end{array}$ & $\begin{array}{l}\text { Seed Yield } \\
\text { (kg/ha) }\end{array}$ & $\begin{array}{c}\text { Haulm } \\
\text { Yield (kg/ } \\
\text { ha) }\end{array}$ & $\begin{array}{c}\text { Harvest } \\
\text { Index } \\
(\%)\end{array}$ \\
\hline $\begin{array}{c}\text { T1 - Imazethapyr } 10 \% \text { SL 500ml/ } \\
\text { ha }\end{array}$ & 46.5 & 3.11 & 12.4 & 16.6 & 9.2 & 34.1 & 645 & 1467 & 30.5 \\
\hline $\begin{array}{c}\text { T2 - Imazethapyr } 10 \% \text { SL } 625 \mathrm{ml} / \\
\text { ha }\end{array}$ & 48.7 & 3.39 & 14.2 & 21.2 & 9.2 & 34.7 & 804 & 1674 & 32.4 \\
\hline $\begin{array}{c}\text { T3 - Imazethapyr } 10 \% \text { SL } 750 \mathrm{ml} / \\
\text { ha }\end{array}$ & 48.6 & 3.42 & 14.4 & 27.4 & 10.1 & 34.4 & 925 & 1876 & 33 \\
\hline $\begin{array}{l}\text { T4 - Imazethapyr } 10 \% \text { SL } 500 \\
\text { ml/ha + Adjuvant @ } 2.0 \mathrm{ml} / 1 \text { of } \\
\text { water }\end{array}$ & 45.8 & 3.16 & 13.1 & 20.2 & 9.3 & 34.3 & 782 & 1678 & 31.8 \\
\hline $\begin{array}{c}\text { T5 - Imazethapyr } 10 \% \text { SL } 625 \\
\text { ml/ha + Adjuvant @ 2.0ml/l of } \\
\text { water }\end{array}$ & 48.9 & 3.19 & 14.3 & 25.5 & 10.4 & 34.6 & 920 & 1903 & 32.6 \\
\hline
\end{tabular}


Annals of Reviews and Research

\begin{tabular}{|c|c|c|c|c|c|c|c|c|c|}
\hline $\begin{array}{c}\text { T6 - Imazethapyr } 10 \% \text { SL } 750 \\
\text { ml/ha + Adjuvant @ } 2.0 \mathrm{ml} / 1 \text { of } \\
\text { water }\end{array}$ & 49.5 & 3.51 & 14.7 & 29.2 & 10.7 & 34.4 & 1015 & 2013 & 33.5 \\
\hline $\begin{array}{c}\text { T7 - Quizalofop - Ethyl } 5 \text { \% EC } \\
@ 1000 \mathrm{ml} / \mathrm{ha}\end{array}$ & 44.8 & 3.13 & 13 & 22.2 & 10.7 & 34.2 & 785 & 1756 & 30.9 \\
\hline T8 - Standrad check (2 IC+2HW) & 52.7 & 3.53 & 15.4 & 29.4 & 9.6 & 35.1 & 1057 & 2213 & 32.3 \\
\hline T9 - Weedy check & 36.7 & 1.61 & 6.3 & 11.4 & 10.7 & 34 & 567 & 1345 & 29.7 \\
\hline T10 - Weed free & 44.3 & 3.33 & 15.1 & 25.3 & 8.2 & 33.9 & 1108 & 1924 & 36.5 \\
\hline S. Em. \pm & 1.1 & 0.4 & 0.8 & 0.4 & 0.1 & 0.91 & 72 & 81 & - \\
\hline$C D(p=0.05)$ & 3.3 & 1.3 & 2.5 & 1.3 & 0.4 & NS & 191 & 209 & - \\
\hline
\end{tabular}

The yield attributing characters of greengram viz., number of pods per plant and number of grains/pods were higher under weed free check and standard check on pooled basis (Table 5). Among the herbicide doses, imazethapyr application of $750 \mathrm{ml} /$ ha produced significantly higher number of pods/plant and number of seeds/pod than that of lower doses of application. It could be due better control of weed might have encouraged the crop to perform better and was attributed by significantly taller plants, higher LAI and total dry matter production [10]. Lower weed controlling efficiencies and higher weed growth under low doses of imazethapyr application might have lead to poor growth of plants which in turn caused lesser yield attributing characters. Application of imazethapyr at $750 \mathrm{ml} / \mathrm{ha}+2 \mathrm{ml}$ adjuvant per liter of water registered the significantly higher seed $1015 \mathrm{~kg} / \mathrm{ha}$ and haulm $2013 \mathrm{~kg} / \mathrm{ha}$ yields and was comparable with that of Imazethapyr $10 \%$ SL 750ml/ha alone and Imazethapyr $10 \%$ SL $625 \mathrm{ml} / \mathrm{ha}+2 \mathrm{ml}$ adjuvant per liter of water. The lowest seed yield of $567 \mathrm{~kg} / \mathrm{ha}$ was recorded by weedy check and this was significantly lower than rest of the treatments. Superior yield attributing characters and effective weed control contributed to higher yields under higher doses of early post emergence application of Imazethapyr $10 \%$ SL 750ml/ha + Adjuvant @ 2.0ml/l of water. These findings are in accordance with the findings of [6] and [11].

\section{Conclusion}

From the current study it was noticed observed that, application of imazethapyr at $750 \mathrm{ml} / \mathrm{ha}+2 \mathrm{ml}$ adjuvant per liter of water found superior in managing weeds of rainfed greengram by recording significantly superior weed control efficiency, higher growth, yield attributes, seed and stover yield.

\section{References}

1. Chaturvedi S K, Ali M (2002) Poor man's meat needs fresh fillip. The Hindu Survey of Indian Agric 63-69.

2. Shuaib OSB (2001) Critical period for weed competition in green gram Univ. Aden J Natural App Sci 5: 11-18.

3. Dungarwal HS, Chaplot PC, Nagda BL (2003) Chemical weed control in mungbean (Plaseolus radiatus L). Indian J Weed Sci 35: 283-284.

4. Anonymous (2012) Package of practice for cultivation of field crops. University of Agricultural Sciences, Dharwad, India.

5. Goud VV, Murade NB, Khakre MS, Patil AN (2013) Efficacy of imazethapyr and quizalofop-ethyl herbicides on growth and yield of chickpea. The Bioscan 8(3): 1015-1018.

6. Tiwari DK, Kewat ML, Khan JA, Khamparia (2007) Evaluation of efficacy of post emergence herbicides in soybean (Glycine max). Indian J Agron 52(1): 74-76.

7. Mishra JS, Chandra Bhanu C (2006) Effect of herbicides on weeds, nodulation and growth of rhizobium in summer blackgram (Vigna mungo). Indian J Weed Sci 38(1\&2): 150-153.

8. Richburge JS, Wilcut JW, Vencill WK (1996) Imazethapyr systems for peanut (Arachis hypogaea L.). Peanut Sci 23(1): 9-14.

9. Kundu R, Bera PS, Brahmachari K (2009) Effect of different weed management practices in summer mungbean [Vigna radiata L.] under new alluvial zone of West Bengal. J Crop Weed 5(2): 117-121.

10. Veeraputhiran R, Chinnusamy C (2008) Performance of time and dose of post emergence herbicide application on relay cropped black gram. Indian J Weed Sci 40(3\&4): 173-175.

11. Gowsia Begum, Rao AS (2006) Efficacy of herbicides on weeds and relay crop of black gram. Indian J Weed Science 38 (1\&2) :145-147. 
(c) (i) This work is licensed under Creative Commons Attribution 4.0 License
Your next submission with Juniper Publishers will reach you the below assets

- Quality Editorial service

- Swift Peer Review

- Reprints availability

- E-prints Service

- Manuscript Podcast for convenient understanding

- Global attainment for your research

- Manuscript accessibility in different formats

( Pdf, E-pub, Full Text, Audio)

- Unceasing customer service

Track the below URL for one-step submission https://juniperpublishers.com/online-submission.php 